ORIGINAL ARTICLE

\title{
Validation of the Paediatric Triage Tape
}

\section{A Wallis, S Carley}

Emerg Med J 2006;23:47-50. doi: 10.1136/emj.2005.024893

\begin{abstract}
Introduction: The Paediatric Triage Tape (PTT) is an easy to use major incident primary triage tool, based upon a modification of the Triage Sieve. The purpose of this study was to prospectively validate the PTT for use in paediatric major incidents. Methods: A database of children presenting the Trauma Unit of the Red Cross Children's Hospital, Cape Town, was developed over a nine month period. Each child was triaged using the PTT, and had an Injury Severity Score (ISS) calculated. Additionally, the New Injury Severity Score (NISS) was calculated, and the presence of interventions that may occur to the children ("Garner criteria") was documented. The sensitivity, specificity, overtriage, and undertriage rates were calculated. Results: 3461 children were entered into the database. For identifying children with an ISS of over 15, the PTT had a sensitivity of $37.8 \%$, specificity of $98.6 \%$, overtriage rate of $38.8 \%$, and an undertriage rate of 3.5\%. Against the NISS and Garner criteria, the results were comparable. Conclusion: The PTT has poor sensitivity at identifying immediate priority children by these criteria.

Specificity (the ability to identify non-T1 patients) is excellent, and the overtriage and undertriage rates are within the range deemed unavoidable by the American College of Surgeons.
\end{abstract}

See end of article for authors' affiliations

Correspondence to: $\operatorname{Dr} L A$ Wallis, PO Box 901, Wellington, 7654, South Africa; leewallis@ bvr.co.za

Accepted for publication 14 May 2005

A lthough originally developed for use in military conflicts, triage is equally applicable to civilian major incidents. It is a key component of medical support during a major incident, ${ }^{1}$ and allows an unmanageable task to be divided into component parts. There are numerous triage systems that exist for use on a day to day basis, both prehospital and inhospital. A number of these have been modified to produce triage systems for use in major incidents. ${ }^{12}$ During major incidents, different systems are typically applied for primary and secondary triage. Primary triage is a very rapid "first look", quickly categorising patients by simple discriminators. The simplest and fastest systems tend to be based on easy to identify parameters that can be detected by personnel with any degree of training. For example, in many systems the ability to walk leads to automatic triage as T3. In addition to such items, physiological parameters are typically used in primary and secondary triage schemes, as they are reproducible to measure and are not dependant upon operator experience. Such physiology generally involves respiratory rate and heart rate, although capillary refill time is occasionally advocated (as in the Triage Sieve ${ }^{1}$ ).

Whichever triage system is used, all healthcare resources at the scene must use it. Furthermore, the system must be easy to teach (so that inexperienced personnel can quickly adopt it and use it at the scene), fast to perform, and accurate (it must identify those patients who are seriously injured as well as those who are less so).

There are specific concerns about the triage of children in major incidents: these have often been raised in major incident case reports ${ }^{3}$ and it is a commonly expressed concern on major incident management courses. ${ }^{1}$ These concerns have been directed at the effectiveness of adult based triage tools to accurately triage children. Most major incident triage systems are based on adult physiology: if these values are applied to small children then there will be an artificially high triage priority assigned. Although this may be thought of as a useful thing (so that children are removed from the scene at the earliest opportunity), it is likely that paediatric resources (both at the scene and at hospital) will be limited and will risk becoming overwhelmed by

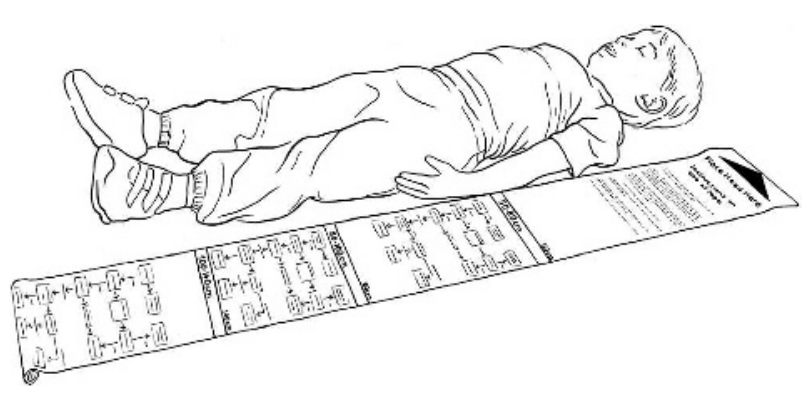

Figure 1 The Paediatric Triage Tape. The tape is placed next to the child from the head end. The algorithm next to the child's feet is then used to triage the child.

inappropriately triaged children. This can lead to genuine cases not receiving the care that they require: Frykberg showed a clear relation between increasing overtriage and increasing mortality. ${ }^{5}$

In order to help overcome this problem, a child specific major incident triage tool is needed. One such tool that is currently available is the Paediatric Triage Tape (PTT) ${ }^{6}$ (see fig 1), in use throughout the UK and many other countries. It is a vinyl waterproof tape, derived from, and using exactly the same flow process as, the Triage Sieve. It has specific triage blocks for children measuring $<50 \mathrm{~cm}, 50-80 \mathrm{~cm}$, $80-100 \mathrm{~cm}, 100-140 \mathrm{~cm}$, and $>140 \mathrm{~cm}$. It has not yet been validated.

Two other specific paediatric primary triage tools are in common use: JumpSTART ${ }^{7}$ methodology is used throughout much of the United States, while Careflight ${ }^{8}$ is used in many parts of Australia. Neither tool has been validated for use in children, and there is no evidence which of these tools is the best to use in a major incident.

Abbreviations: ACSCOT, American College of Surgeons Committee on Trauma; ISS, Injury Severity Score; NISS, New Injury Severity Score; PTT, Paediatric Triage Tape. 


\section{Table 1 Garner criteria}

\begin{tabular}{ll}
\hline $\begin{array}{l}\text { Operative intervention } \\
\text { Fluid resuscitation }\end{array}$ & $\begin{array}{l}\text { (Non-orthopaedic; within 6 hours) } \\
(1000 \mathrm{ml} \text { or more, to maintain BP } \\
>89 \mathrm{mmHg})\end{array}$ \\
$\begin{array}{l}\text { Invasive CNS monitoring } \\
\text { A procedure to maintain the }\end{array}$ & $\begin{array}{l}\text { (Or a positive head CT scan) } \\
\text { airway }\end{array}$ \\
$\begin{array}{l}\text { Decompression of a tension } \\
\text { pneumothorax }\end{array}$ &
\end{tabular}

\section{Validation of triage tools}

Triage tools are traditionally validated against the Injury Severity Score $\left(\right.$ ISS $\left.^{9}\right)$. However, the New Injury Severity Score (NISS) ${ }^{10}$ may be more accurate as it measures the three worst injuries in any body region, rather than using the highest score in each of three different body regions. ${ }^{11}$ Whichever of the two systems is chosen, there are limitations in applying it against major incident triage algorithms: both tools are only designed to identify major trauma patients (those who are Tl (immediate), or not-T1) and have no discriminatory value between T2 (urgent) and T3 (delayed) patients; they have only been shown to accurately predict death; they focus on injury pattern and not the requirement for medical intervention (the main drive behind triage at a major incident); and they are of no help in assessing the triage of non-injured (medical) patients.

An alternative solution has been proposed by Baxt and Upnekies, ${ }^{12}$ later modified by Garner $e t a l^{13}$ to be applicable to major incident situations: they proposed that specific interventions rather than injury scores should be used as the determinants of outcome and hence triage category. The criteria used by Garner et al are shown in table 1.

Although these criteria were designed for comparison of adult major incident triage schemes, they may be applied (with simple modification) to children. The requirement for fluid resuscitation $(>1000 \mathrm{ml})$ may be modified to the requirement for resuscitation of over $20 \mathrm{ml} / \mathrm{kg}$ of fluid (in excess of the first fluid bolus recommended by the Advanced Paediatric Life Support Course $\mathrm{C}^{14}$ ).

For the purposes of this article, the validation of the PTT will occur against the ISS, with concurrent comparison to NISS and Garner criteria (modified for children, but simply referred to as modified Garner criteria throughout this article). The aim of this article is to validate the PTT as a major incident primary triage tool.

\section{METHODS}

The Trauma Unit of the Red Cross Children's Hospital, Cape Town, sees children aged up to 12 years. As the major tertiary referral centre for the Cape Town area, it receives approximately 9000 injured children each year. Over nine months of 2002, a prospective database of attendees was compiled, as the basis for the validation of the PTT.

Children were considered eligible for enrolment in the study if they were aged under 13 years and presented within 12 hours of an acute injury.

\begin{tabular}{|c|c|c|c|c|}
\hline & & \multicolumn{2}{|c|}{ PTT triage } & \multirow[b]{2}{*}{ Total } \\
\hline & & Tl & Not-Tl & \\
\hline \multirow[t]{3}{*}{ ISS } & $16+$ & 71 & 117 & 188 \\
\hline & $<16$ & 47 & 3226 & 3273 \\
\hline & Total & 118 & 3343 & 3461 \\
\hline \multirow[t]{3}{*}{ NISS } & $16+$ & 82 & 232 & 314 \\
\hline & $<16$ & 36 & 3111 & 3147 \\
\hline & Total & 118 & 3343 & 3461 \\
\hline \multirow[t]{3}{*}{ Garner } & Yes & 83 & 117 & 200 \\
\hline & No & 35 & 3226 & 3261 \\
\hline & Total & 118 & 3343 & 3461 \\
\hline
\end{tabular}

Children were triaged on arrival at the Trauma Unit, using the PTT, as T1, T2, T3, or Dead using either their initial assessment in the trauma unit, or data as recorded on arrival at the scene if brought by paramedics. All children were prospectively followed through to death or discharge. Each child had an ISS and NISS determined. Data were recorded concerning the presence of any of the modified Garner criteria.

The sensitivity, specificity, overtriage, and undertriage rates of the PTT were calculated against ISS, NISS, and modified Garner criteria. Sensitivity reflects the proportions of those patients who are $\mathrm{Tl}$ who are correctly identified as $\mathrm{Tl}$; specificity is the proportion of patients who are not-Tl who are correctly identified as not-Tl; the overtriage rate represents the proportion of patients who are triaged Tl but are not- $\mathrm{Tl}$, and undertriage represents the patients who are identified as not-Tl who actually are $\mathrm{Tl}$.

\section{RESULTS}

In the study period, 5508 children presented to the Trauma Unit within 12 hours of injury. Of these, 3597 children met the entry criteria for the study: 3461 (96\%) children were enrolled onto the database (see fig 2). Sixty three per cent were male, with a median age of 7 years.

There were $188(5.4 \%)$ with an ISS of $16+, 314(9.1 \%)$ with NISS 16+, and 312 Garner criteria were present in 200 (5.8\%) children. For each of these standards, the sensitivity, specificity, overtriage, and undertriage rates for the PTT overall are presented in table 2 . The primary data from which these calculations were made is shown in table 3 . Table 4 shows the breakdown of these results for each height block.

Only five children were under $50 \mathrm{~cm}$, and all were triaged $\mathrm{Tl}$ in accordance with the PTT's instructions. All had an ISS under 16, NISS under 16, and none had any Garner criteria present.

\section{DISCUSSION}

An ideal triage tool will correctly spot all Tl patients (high sensitivity); however, the higher the sensitivity the lower the specificity (the ability to correctly spot patients who are notT1) resulting in more patients being directed for immediate care, and risking swamping medical resources. In a major incident, a high degree of specificity is essential.

\begin{tabular}{lclllll} 
Table 2 & PTT: results by ISS, NISS, and presence of one or more Garner criteria \\
\hline & ISS (\%) & $95 \% \mathrm{Cl}$ & NISS (\%) & $\mathbf{9 5 \% ~ C l}$ & Garner (\%) & $\mathbf{9 5 \% ~ C l}$ \\
\hline Sensitivity & 37.8 & $32.7-42.5$ & 26.1 & $23-28.8$ & 41.5 & $36.8-45.6$ \\
Specificity & 98.6 & $98.3-98.8$ & 98.9 & $98.5-99.1$ & 98.9 & $98.6-99.2$ \\
Overtriage & 38.8 & $32.3-47.9$ & 30.5 & $23.2-38.8$ & 29.5 & $22.7-37.5$ \\
Undertriage & 3.5 & $3.2-3.8$ & 6.9 & $6.7-7.2$ & 3.5 & $3.3-3.8$ \\
\hline
\end{tabular}




\begin{tabular}{|c|c|c|c|c|c|c|c|}
\hline $\begin{array}{l}\text { Table } 4 \\
\text { criteria }\end{array}$ & \multicolumn{7}{|c|}{ Height blocks of PTT: results by ISS, NISS, and presence of one or more Garner } \\
\hline \multicolumn{2}{|c|}{ Height block $(\mathrm{cm})$} & \multirow{2}{*}{$\begin{array}{l}\text { ISS (\%) } \\
21.7\end{array}$} & \multirow{2}{*}{$\begin{array}{l}95 \% \mathrm{Cl} \\
11.3-27.9\end{array}$} & \multirow{2}{*}{$\frac{\text { NISS (\%) }}{16.1}$} & \multirow{2}{*}{$\frac{95 \% \mathrm{Cl}}{8.3-20.7}$} & \multirow{2}{*}{$\begin{array}{l}\text { Garner (\%) } \\
25\end{array}$} & \multirow{2}{*}{$\begin{array}{l}95 \% \mathrm{Cl} \\
13-32.1\end{array}$} \\
\hline $50-80$ & Sensitivity & & & & & & \\
\hline & Specificity & 99.6 & $99.1-99.9$ & 99.6 & $99.1-99.9$ & 99.6 & $99.1-99.9$ \\
\hline & Overtriage & 28.6 & $8.4-62.9$ & 28.6 & $8.3-63.3$ & 28.6 & $8.4-62.8$ \\
\hline & Undertriage & 3.4 & $3.2-3.9$ & 5 & $4.7-5.4$ & 2.9 & $2.6-3.3$ \\
\hline \multirow[t]{4}{*}{$80-100$} & Sensitivity & 20 & $12-27.6$ & 19 & $12.4-24.1$ & 23.6 & $16.6-27.9$ \\
\hline & Specificity & 99 & $98.6-99.4$ & 99.3 & $98.8-99.6$ & 99.5 & $99-99.8$ \\
\hline & Overtriage & 47.1 & $27-67.2$ & 35.3 & $17.7-57.8$ & 23.5 & $9.7-46.2$ \\
\hline & Undertriage & 3.3 & $3.9-4.7$ & 5.6 & $5.2-6.0$ & 5 & $4.7-5.4$ \\
\hline \multirow[t]{4}{*}{$100-140$} & Sensitivity & 50.6 & $42.4-57.9$ & 30.5 & $26.1-34$ & 54.7 & $47.4-60.8$ \\
\hline & Specificity & 98.2 & $97.8-98.6$ & 98.7 & $98.2-99.1$ & 98.7 & $98.3-99.1$ \\
\hline & Overtriage & 38.9 & $30-42.8$ & 26.4 & $17.9-36.8$ & 27.8 & $19.8-37.4$ \\
\hline & Undertriage & 2.7 & $2.3-3.2$ & 7.56 & $7.2-8.1$ & 2.7 & $2.3-3.1$ \\
\hline \multirow[t]{4}{*}{$>140$} & Sensitivity & 39.4 & $28.2-46.4$ & 25.5 & $18-30.1$ & 43.3 & $31.2-51$ \\
\hline & Specificity & 98.9 & $97.9-99.5$ & 98.9 & $97.8-99.5$ & 98.9 & $98-99.5$ \\
\hline & Overtriage & 23.5 & $8.9-45.2$ & 23.5 & $9.7-46$ & 23.5 & $10-44.5$ \\
\hline & Undertriage & 5.2 & $4.6-6.1$ & 9.8 & $9.2-10.8$ & 4.4 & $3.8-5.3$ \\
\hline
\end{tabular}

The American College of Surgeons Committee on Trauma (ACSCOT) states that triage tools will have an unavoidable undertriage rate of $5-10 \%$, associated with an overtriage rate of $30-50 \% .{ }^{15}$ Excessive overtriage of children may be seen to be beneficial, to quickly remove them from the scene, but risks swamping limited paediatric resources. Undertriage results in missing those children who need immediate care: both undertriage and overtriage must be minimised.

\section{Principal findings}

The PTT was found to have poor sensitivity against ISS, NISS, and Garner criteria, indicating that it does not detect all of the Tl patients. Part of the explanation for this may lie in the very high specificity (up to $98.9 \%$ ): the PTT is excellent at identifying patients who are not-T1, but poor at identifying those who are $\mathrm{Tl}$. The undertriage and overtriage rates against all three gold standards were within the recommendations of the ACSCOT report. ${ }^{15}$

No height block was more predictive than the PTT overall. Only five children were under $50 \mathrm{~cm}$ in length, and all were triaged T1. It is unlikely that a large enough sample of injured children of this size will ever be achieved in order to make meaningful conclusions (as they are unlikely to be out of hospital at this size). However, the current practice of triaging these children as $\mathrm{Tl}^{1}$ is likely to be safe as it removes this difficult group from the scene rapidly, and represents a tiny proportion of any likely patient load that will be potentially overtriaged.

\section{Strengths and weaknesses of the study}

A 12 hour cut off for entry into the study may be considered to be excessively long (although there are no data concerning the time to presentation for casualties in major incidents in the UK). Although this may be true in areas with rapid access to and assessment of casualties, there is no guarantee that this will be the only place in which the PTT has the potential to be used. It is well known that casualties in larger major incidents, remote incidents, and in incidents occurring in less well developed countries (for example in South Africa) that the time from injury to assessment will be longer than is typically the case in normal day to day European practice. We therefore chose the time limit of 12 hours as an arbitrary cut off that we felt represented patients who attended hospital as a result of their primary injury rather than as a result of a secondary deterioration from an initial injury.

Although this study was designed to prospectively assess the utility of the PTT, the number of patients enrolled with an ISS of $15+$ is relatively small, especially in some of the height blocks, producing results with wide confidence intervals. However, as the majority of patients from a major incident are likely to be minor in nature, ${ }^{16}$ the patient distribution in this study is representative.

Choosing to validate a UK based triage system in a developing country may lead to bias in the conclusions, as the physiological parameters used by the tool may be different in that country. Work undertaken by one of the authors (LAW, in press) shows that the heart rate and respiratory rate of children in the UK and South Africa are identical by height or weight (although not by age, as children in South Africa are smaller and less heavy at any given age). Hence, direct extrapolation of the results to the UK population is possible.

One of the gold standards chosen for this study (Garner criteria) was developed as expert opinion, and has not been validated as an outcome measure for a triage tool. Furthermore, these criteria were developed to test adult based triage tools. ${ }^{17}$ However, they highlight some of the common interventions that a patient may require following injury in a major incident, and these criteria may equally be applied to children as well as adults.

The ability to identify patients as T2, T3, or dead cannot be measured with current gold standards.

Strengths and weaknesses in relation to other studies There have been no other studies attempting to validate major incident primary triage algorithms in children. However, Garner et al reported much higher sensitivities in their analysis of adult primary triage tools $(82-85 \%) \cdot{ }^{17}$

\section{Meaning of the study}

The PTT is known to be easy to learn, fast, and easy to use: ${ }^{6}$ these properties are very favourable in major incident primary triage algorithms. This study shows that the PTT is a poor tool to identify seriously injured children (although the utility of this outcome in a major incident setting is of doubt).

\section{Unanswered questions and future research}

Whichever triage algorithm is used, it should be validated in conditions as close as possible to those in which it is used. This is unlikely to ever occur in the setting of a major incident, due to the very nature of these incidents. Computer modelling and major incident registries may help in this regard in the future. However, the only widely accepted gold standard currently available for testing triage algorithms is the ISS. The NISS is felt by many to be superior to the ISS as 
it incorporates data from the three worst injuries sustained: ${ }^{11}$ it is yet to gain wide acceptance as either a triage algorithm validation tool or a trauma system analysis tool (the main current use of ISS).

The use of an ISS of 16 or higher as a marker of major trauma is well established in regionalised systems of health care such as the USA. As this level of ISS is associated with worse outcomes, it is appropriate to use ISS $16+$ as a marker of those patients who should be identified as immediate (Tl) in a major incident setting. The same argument holds well for NISS of 16+. However, the group of patients with an ISS (or NISS) of 14 or below may contain some people who should be triaged as T2 (urgent) and some T3 (delayed). Neither ISS nor NISS allows for differentiation between these groups. For this reason, it is appropriate to use ISS/NISS 16+ as a marker of immediate priority, but an ISS of 15 and below is of no discriminatory value.

Furthermore, in a major incident it is not the severity of specific injuries that is of importance when undertaking primary triage, but rather the requirement for medical intervention. The ISS/NISS are not measuring outcomes that are helpful in major incident setting. This problem is overcome by the use of Garner criteria, which identify patients in need of specific interventions. However, like ISS/ NISS these criteria may only be used to identify those patients to be triaged Tl (immediate), with no discriminatory value for not-Tl patients.

Altering the physiological parameters used on the PTT to identify a change in triage category is an essential next step. This is currently being researched in Cape Town and is likely to require modification of the respiratory rate and heart rate values for each of the height blocks. Once this analysis is completed the new algorithm may then be prospectively validated.

\section{CONCLUSION}

The PTT is a simple to use tool for primary triage at major incidents. As it is based upon the Triage Sieve, it should be easy for most practitioners in the UK to become familiar with its use.

It has good undertriage and overtriage rates, and excellent specificity although sensitivity is poor (it only correctly identifies less than half of all $\mathrm{Tl}$ patients, meaning that many seriously injured children will not be detected by this tool). The reason behind this may lie in the ranges of physiological values at which triage category changes. Work is currently underway to modify these values in order to increase the sensitivity of the PTT, although it is accepted that this will inevitably be at the expense of less specificity.
In the meantime, in areas where the PTT is currently used we recommend its ongoing usage pending redesign, as any problems with sensitivity may be outweighed by the problems of learning a new tool.

\section{Authors' affiliations}

L A Wallis, Consultant in Paediatric Emergency Medicine, Red Cross Children's Hospital, Cape Town, South Africa

S Carley, Consultant in Emergency Medicine, Manchester Royal Infirmary, Manchester, UK

Funding: none.

Competing interests: none.

\section{REFERENCES}

1 Advanced Life Support Group. Major incident medical management and support: the practical approach. London: BMJ Books, 2002.

2 Champion HR, Sacco WJ, Copes WS, et al. A revision of the Trauma Score. J Trauma 1989;29:623-9.

3 Vukmir RB, Paris PM. The Three Rivers Regatta accident: an EMS perspective. Am J Emerg Med 1991;9:64-71.

4 van Amerongen RH, Fine JS, Tunik MG, et al. The Avianca plane crash: an emergency medical system's response to pediatric survivors of the disaster. Pediatrics 1993:92:105-10.

5 Frykberg ER. Medical management of disasters and mass casualties from terrorist bombings: how can we cope? J Trauma 2002;53:201-12.

6 Hodgetts TJ, Hall J, Maconochie I, et al. Paediatric Triage Tape. Pre-Hospital Immediate Care 1998;2:155-9.

7 Romig LE. Pediatric triage. A system to JumpSTART your triage of young patients at MCls. JEMS 2002;27:52-8, 60-3.

8 Nocera A, Garner A. An Australian mass casualty incident triage system for the future based upon triage mistakes of the past: the Homebush Triage Standard. Aust N Z J Surg 1999;69:603-8.

9 Baker SP, O'Neill B, Haddon W Jr, et al. The injury severity score: a method for describing patients with multiple injuries and evaluating emergency care. J Trauma 1974;14:187-96.

10 Osler T, Baker SP, Long W. A modification of the injury severity score that both improves accuracy and simplifies scoring. J Trauma 1997;43:922-5, discussion 925-6.

11 Lavoie A, Moore L, LeSage N, et al. The New Injury Severity Score: a more accurate predictor of in-hospital mortality than the Injury Severity Score. J Trauma 2004;56:1312-20.

12 Baxt WG, Upenieks V. The lack of full correlation between the Injury Severity Score and the resource needs of injured patients. Ann Emerg Med 1990;19:1396-400.

13 Garner A, Lee A, Harrison K, et al. Comparative analysis of multiple-casualty incident triage algorithms. Ann Emerg Med 2001;38:541-8.

14 Advanced Life Support Group. Advanced paediatric life support: the practical approach. London: BMJ Books, 2005.

15 American College of Surgeons Committee on Trauma. Resources for optimal care of the injured patient: 1999. Chicago: American College of Surgeons, 1998.

16 Carley SD, Mackway-Jones K. The casualty profile from the Manchester bombing 1996: a proposal for the construction and dissemination of casualty profiles from major incidents. J Accid Emerg Med 1997;14:76-80.

17 Garner A, Lee A, Harrison K, et al. Comparative analysis of multiple-casualty incident triage algorithms. Ann Emerg Med 2001;38:541-8. 